\title{
Traffic Parameters Estimation to Predict Road Side Pollutant Concentrations using Neural Networks
}

\author{
Fabio Galatioto • Pietro Zito • Marco Migliore
}

Published online: 21 July 2009

(C) Springer Science + Business Media B.V. 2009

\section{Erratum to: Environ Model Assess \\ DOI 10.1007/s10666-007-9129-z}

The original version of this article unfortunately contained a mistake. Marco Migliore was not listed among the authors.

The article was published in Volume 14 Issue 3 pp. 365-374.

The online version of the original article can be found at http://dx.doi. org/10.1007/s10666-007-9129-z.

F. Galatioto $\cdot$ P. Zito $\cdot$ M. Migliore

Department of Transportation Engineering, University of Palermo,

Viale delle Scienze, Edificio n. 8,

90128 Palermo, Italy

F. Galatioto $\cdot$ P. Zito $(\varangle) \cdot$ M. Migliore

Dipartimento di Ingegneria dei Trasporti,

Università degli Studi di Palermo,

Viale delle Scienze, Edificio n. 8,

90128 Palermo, Italy

e-mail: zito@ditra.unipa.it 